\title{
MIR373 Pre-miRNA
}

National Cancer Institute

\section{Source}

National Cancer Institute. MIR373 Pre-miRNA. NCI Thesaurus. Code C82826.

MIR373 is an oligoribonucleotide that is encoded by the human MIR373 gene and has a role in the regulation of gene expression. 